\title{
Explicit integration of a generic Hénon-Heiles system with quartic potential
}

Nicola Sottocornola

To cite this article: Nicola Sottocornola (2017) Explicit integration of a generic HénonHeiles system with quartic potential, Journal of Nonlinear Mathematical Physics 24:3, 346-355, DOI: https://doi.org/10.1080/14029251.2017.1341697

To link to this article: https://doi.org/10.1080/14029251.2017.1341697

Published online: 04 January 2021 


\title{
Explicit integration of a generic Hénon-Heiles system with quartic potential
}

\author{
Nicola Sottocornola \\ Department of Mathematics and Statistics \\ Zayed University, Abu Dhabi, UAE \\ nicola.spinelli@zu.uc.ae
}

Received 28 February 2017

Accepted 30 March 2017

\begin{abstract}
There are seven time independent, integrable, Hénon-Heiles systems: three with cubic and four with quartic potential. The cubic and one of the quartic cases have been separated in the last decades. The other three cases 1:6:1, 1:6:8 and 1:12:16 have resisted several attempts in the last years. In this paper we focus our attention on the 1:12:16 case whose equations of motion have been separated only in the degenerate case $a b=0$. We give here the separation coordinates for the generic case using a method introduced by Franco Magri in 2005 under the name of Kowalevski's Conditions.
\end{abstract}

Keywords: Integrable systems; separation of coordinates; Hénon-Heiles systems.

2000 Mathematics Subject Classification: 37J35, 34M55

\section{Introduction}

We consider, in the symplectic space $\mathbb{R}^{4}$ endowed with the standard symplectic structure

$$
d p_{1} \wedge d x+d p_{2} \wedge d y
$$

the Hamiltonians $H$ of the form

$$
H=\frac{1}{2}\left(p_{1}^{2}+p_{2}^{2}\right)+V(x, y)
$$

These systems pass the Painlevé test for 7 polynomial functions $V: 3$ cubic and 4 quartic. Some extra non-polynomial terms can be added without destroying the integrability, giving rise to the so called generalized versions. The cubic cases, called SK, KdV5, KK because of their links with some well-known ODEs, have been separated decades ago.

- KdV5. This system can be separated in different ways, for instance with parabolic coordinates [12]

$$
\begin{aligned}
& h_{1}=\frac{1}{2}\left(p_{1}^{2}+p_{2}^{2}+\omega_{1} x^{2}+\omega_{2} y^{2}\right)+a x y^{2}+2 a x^{3}+\frac{b}{2 y^{2}} \\
& h_{2}=4 a p_{2}\left(y p_{1}-x p_{2}\right)+\left(4 \omega_{2}-\omega_{1}\right)\left(\omega_{2} y^{2}+p_{2}^{2}+\frac{b}{y^{2}}\right)+a^{2} y^{2}\left(4 x^{2}+y^{2}\right)+4 a x\left(\omega_{2} y^{2}-\frac{b}{y^{2}}\right)
\end{aligned}
$$


N. Sottocornola / Explicit integration of a generic Hénon-Heiles system. . .

- SK. This case and the next one have been separated and integrated with hyperelliptic functions [11]

$$
\begin{aligned}
& h_{1}=\frac{1}{2}\left(p_{1}^{2}+p_{2}^{2}\right)+\frac{1}{2} \omega\left(x^{2}+y^{2}\right)+a x y^{2}+\frac{1}{3} a x^{3}-\frac{b^{2}}{2 y^{2}} \\
& h_{2}=\left(3 a x^{2} y+a y^{3}+3 \omega x y+3 p_{1} p_{2}\right)^{2}-3 b^{2}\left(3 \frac{p_{1}^{2}}{y^{2}}+4 a x+2 \omega^{2}\right)
\end{aligned}
$$

- KK.

$$
\begin{aligned}
h_{1}= & \frac{1}{2}\left(p_{1}^{2}+p_{2}^{2}\right)+8 \omega x^{2}+\frac{1}{2} \omega y^{2}+a x y^{2}+\frac{16}{3} a x^{3}+\frac{1}{2} \frac{b}{y^{2}} \\
h_{2}= & \left(3 \omega y^{2}+3 p_{2}^{2}+3 \frac{b}{y^{2}}\right)^{2}+12 a p_{2} y^{2}\left(-p_{1} y+3 p_{2} x\right)-2 a^{2} y^{4}\left(6 x^{2}+y^{2}\right) \\
& +12 a x\left(-\omega y^{4}+b\right)-12 \omega b
\end{aligned}
$$

The 4 cases with quartic potential are called 1:2:1, 1:6:1, 1:6:8 and 1:12:16 according to the ratios of the coefficients of the quartic terms of the potential function:

- 1:2:1. It can be integrated with a canonical transformation to elliptical coordinates, reducing the problem to an ODE which is similar to the one in the KdV5 case [13]

$$
\begin{aligned}
& h_{1}=\frac{1}{2}\left(p_{1}^{2}+p_{2}^{2}\right)+\frac{1}{2} \omega_{1} x^{2}+\frac{1}{2} \omega_{2} y^{2}+\frac{1}{2}\left(x^{2}+y^{2}\right)^{2}-\frac{1}{2} \frac{a^{2}}{x^{2}}-\frac{1}{2} \frac{b^{2}}{y^{2}} \\
& h_{2}=\left(p_{1} y-p_{2} x\right)^{2}-y^{2} \frac{a^{2}}{x^{2}}-x^{2} \frac{b^{2}}{y^{2}}-\frac{1}{2}\left(\omega_{1}-\omega_{2}\right)\left(x^{4}-y^{4}+\omega_{1} x^{2}-\omega_{2} y^{2}+p_{1}^{2}-p_{2}^{2}-\frac{a^{2}}{x^{2}}+\frac{b^{2}}{y^{2}}\right)
\end{aligned}
$$

- 1:6:1. This system has been separated only in the case $a^{2}=b^{2}[1]$ :

$$
\begin{aligned}
& h_{1}=\frac{1}{2}\left(p_{1}^{2}+p_{2}^{2}\right)-\frac{1}{2} \omega\left(x^{2}+y^{2}\right)-\frac{1}{32}\left(x^{4}+6 x^{2} y^{2}+y^{4}\right)-\frac{a^{2}}{2 x^{2}}-\frac{b^{2}}{2 y^{2}} \\
& h_{2}=\left(p_{1} p_{2}+x y\left(-\frac{1}{8} x^{2}-\frac{1}{8} y^{2}-\omega\right)\right)^{2}-a^{2}\left(\frac{p_{2}^{2}}{x^{2}}-\frac{1}{4} y^{2}\right)-b^{2}\left(\frac{p_{1}^{2}}{y^{2}}-\frac{1}{4} x^{2}\right)+\frac{a^{2} b^{2}}{x^{2} y^{2}}
\end{aligned}
$$

- 1:6:8. Separated only in the case $a b=0$ [8]:

$$
\begin{aligned}
h_{1}= & \frac{1}{2}\left(p_{1}^{2}+p_{2}^{2}\right)-\frac{1}{2} \omega\left(4 x^{2}+y^{2}\right)-\frac{1}{2} x^{4}-\frac{3}{8} x^{2} y^{2}-\frac{1}{16} y^{4}-a x+\frac{b}{2 y^{2}} \\
h_{2}= & \left(p_{2}^{2}-\frac{1}{8} y^{2}\left(2 x^{2}+y^{2}+8 \omega\right)+\frac{b}{y^{2}}\right)^{2}-\frac{1}{4} y^{2}\left(y p_{1}-2 x p_{2}\right)^{2} \\
& -a\left(2 a y^{2}-4 y p_{1} p_{2}+\frac{1}{2} x y^{4}+x^{3} y^{2}+4 x p_{2}^{2}+4 \omega x y^{2}+4 b \frac{x}{y^{2}}\right)
\end{aligned}
$$

- $1: 12: 16$, Separated only in the case $a b=0$ [10]:

$$
\begin{aligned}
& h_{1}=\frac{1}{2}\left(p_{1}^{2}+p_{2}^{2}\right)+\frac{1}{8} \omega\left(4 x^{2}+y^{2}\right)-\frac{1}{2} x^{4}-\frac{3}{8} x^{2} y^{2}-\frac{1}{32} y^{4}-\frac{a^{2}}{2 x^{2}}-\frac{b^{2}}{2 y^{2}} \\
& h_{2}=\left(8 p_{2}\left(p_{1} y-p_{2} x\right)-x y^{4}-2 x^{3} y^{2}+2 \omega x y^{2}+8 b^{2} \frac{x}{y^{2}}\right)^{2}+16 a^{2}\left(y^{4}-4 \frac{p_{2}^{2} y^{2}}{x^{2}}\right)
\end{aligned}
$$


All these separation coordinates and the methods to find them are resumed in [3]. The paper ends with the authors asking for the separation coordinates of the three generic remaining cases $1: 6: 1,1: 6: 8$ and 1:12:16.

In this paper we solve one of the three problems asked by Conte, Musette and Verhoeven in 2005 [3] and 2006 [4], namely the 1:12:16 one (see Remark 7.2).

In order to obtain the separation coordinates, we make use of a set of separability conditions, called Kowalevski's Conditions (KCs). They come from the bihamiltonian approach to the theory of integrable systems. In this section and in the next one, we limit ourselves to show how this method works without giving any proof. This method is inspired by the one used by Franco Magri in [6]. The reader interested in understanding the theoretical background of the KCs is invited to read [6], [7] and [9] where more examples can be found.

\section{Kowalevski's conditions}

To present the KCs in their simplest form, in accordance with the needs of this paper, it is sufficient to consider the case of an integrable Hamiltonian system on $\mathbb{R}^{4}$ in the form of a pair of commuting Hamiltonian vector fields $X_{h_{1}}$ and $X_{h_{2}}$. We agree to denote by

$$
\dot{F}=X_{h_{1}}(F) \quad F^{\prime}=X_{h_{2}}(F)
$$

the derivatives of a function $F$ along the given Hamiltonian fields. Our goal is to find the separation coordinates of both $h_{1}$ and $h_{2}$.

To this purpose, we build a $2 \times 2$ matrix

$$
M=\left(\begin{array}{ll}
m_{1} & m_{2} \\
m_{3} & m_{4}
\end{array}\right)
$$

called control matrix, whose eigenvalues are the desired coordinates. It is the object which is submitted to KCs. For the sake of clarity, these conditions are splitted in two sets.

Definition 2.1 (The reduced Kowalevski's Conditions). The matrix $M$ is said to obey the reduced set of Kowalevski's conditions if its entries verify the differential constraints:

$$
\begin{aligned}
& X_{h_{1}}\left(m_{3}\right)=X_{h_{2}}\left(m_{1}\right) \\
& X_{h_{1}}\left(m_{4}\right)=X_{h_{2}}\left(m_{2}\right) .
\end{aligned}
$$

These conditions have a simple geometric meaning, which comes to light when one considers the tensor field of type $(1,1)$

$$
\begin{aligned}
& M X_{h_{1}}=m_{1} X_{h_{1}}+m_{2} X_{h_{2}} \\
& M X_{h_{2}}=m_{3} X_{h_{1}}+m_{4} X_{h_{2}},
\end{aligned}
$$

on the Lagrangian leaves spanned by the vector fields $X_{h_{1}}$ and $X_{h_{2}}$. The reader may check that, if $M$ is torsionless, there is a second commutator on the vector fields tangent to the Lagrangian foliation, 
given by:

$$
[X, Y]_{M}=[M X, Y]+[X, M Y]-M[X, Y] .
$$

After that, the reader is in a position to evaluate the commutator $\left[X_{h_{1}}, X_{h_{2}}\right]_{M}$ and to check that it vanishes if the matrix $M$ obeys the reduced KCs. Thus the intrinsic meaning of the reduced conditions is that the Hamiltonian vector fields $X_{h_{1}}$ and $X_{h_{2}}$ commute with respect to both the standard commutator and the deformed one:

$$
\left[X_{h_{1}}, X_{h_{2}}\right]=0 \quad\left[X_{h_{1}}, X_{h_{2}}\right]_{M}=0 .
$$

Definition 2.2 (The full Kowalevski's Conditions). If the matrix $M^{2}$ obeys as well the reduced Kowalevski's conditions, the matrix $M$ is said to obey the full set of Kowalevski's conditions and its characteristic polynomial $s(u)$ is said to fulfil the Kowalevski's property. So, the Kowalevski's conditions require that the coefficients $m_{1}, \ldots, m_{4}$ verify the four differential constraints:

$$
\begin{aligned}
X_{h_{1}}\left(m_{3}\right) & =X_{h_{2}}\left(m_{1}\right) \\
X_{h_{1}}\left(m_{4}\right) & =X_{h_{2}}\left(m_{2}\right) \\
X_{h_{1}}\left(m_{1} m_{3}+m_{3} m_{4}\right) & =X_{h_{2}}\left(m_{1}^{2}+m_{2} m_{3}\right) \\
X_{h_{1}}\left(m_{2} m_{3}+m_{4}^{2}\right) & =X_{h_{2}}\left(m_{1} m_{2}+m_{2} m_{4}\right) .
\end{aligned}
$$

With this language the main result of the theory of KCs can now be stated in the following form:

Theorem 2.3 (Magri, 2005). Let $s(u)=u^{2}-s_{1} u-s_{2}$ be the characteristic polynomial of $M$ and let $u_{1}$ and $u_{2}$ be its roots.

Assume that:

(1) the four equations (2.1) are verified;

(2) the roots $u_{1}$ and $u_{2}$ are real and almost everywhere distinct;

(3) its coefficients $s_{1}$ and $s_{2}$ commute with respect to the canonical Poisson brackets:

$$
\left\{s_{1}, s_{2}\right\}=0
$$

(4) $s_{1}$ and $s_{2}$ are functionally independent from $h_{1}$ and $h_{2}$ almost everywhere on $\mathbb{R}^{4}$ :

$$
d s_{1} \wedge d s_{2} \wedge d h_{1} \wedge d h_{2} \neq 0 .
$$

Then the roots $u_{1}$ and $u_{2}$ are separation coordinates for both vector fields $X_{h_{1}}$ and $X_{h_{2}}$.

\section{The method of syzygies}

The idea is to use the KCs to construct directly half of the separation coordinates. For what has been said before, this goal is reached if one is able to construct a $2 \times 2$ matrix whose entries verify the four equations (2.1), and whose characteristic polynomial has coefficients $s_{1}=m_{1}+m_{4}$ and 
$s_{2}=m_{2} m_{3}-m_{1} m_{4}$ which are in involution and generically independent of the Hamiltonians. The starting point is the equation

$$
F^{\prime}=\dot{G}
$$

modelling the reduced KCs. This equation is nothing else than a special linear combination of the equations of motion, of the form

$$
\frac{\partial F}{\partial p_{1}} p_{1}{ }^{\prime}+\frac{\partial F}{\partial p_{2}} p_{2}{ }^{\prime}+\frac{\partial F}{\partial x} x^{\prime}+\frac{\partial F}{\partial y} y^{\prime}=\frac{\partial G}{\partial p_{1}} \dot{p}_{1}+\frac{\partial G}{\partial p_{2}} \dot{p}_{2}+\frac{\partial G}{\partial x} \dot{x}+\frac{\partial G}{\partial y} \dot{y}
$$

When the equations of motion are rational functions, it is natural to search solutions of this equation in the field of rational functions. After cancellation of the denominators, one ends up to the search of eight polynomials $\left(A_{1}, \ldots, A_{4}, B_{1}, \ldots, B_{4}\right)$ such that

$$
A_{1} p_{1}{ }^{\prime}+A_{2} p_{2}{ }^{\prime}+A_{3} x^{\prime}+A_{4} y^{\prime}=B_{1} \dot{p_{1}}+B_{2} \dot{p_{2}}+B_{3} \dot{x}+B_{4} \dot{y} .
$$

This equation is a syzygy of the equations of motion, and the solution of this equation is the starting point of the method of syzygies.

Once calculated $F$ and $G$ the next step is to guess the form of the control matrix $M$ whose characteristic polynomial is $s(u)$ (see Theorem 2.3). It seems natural to look first for the entries of the control matrix, $m_{1}, m_{2}, m_{3}$ and $m_{4}$, as linear functions in $F$ and $G$ :

$$
\begin{aligned}
& m_{1}=\alpha_{0}+\alpha_{1} F+\alpha_{2} G \\
& m_{2}=\beta_{0}+\beta_{1} F+\beta_{2} G \\
& m_{3}=\gamma_{0}+\gamma_{1} F+\gamma_{2} G \\
& m_{4}=\delta_{0}+\delta_{1} F+\delta_{2} G
\end{aligned}
$$

where the $\alpha, \beta, \gamma$ and $\delta$ are constants of the motion. Let's see how the method works with the simple example $a=b=0$.

\section{The degenerate case $a=b=0$}

Replacing $a=b=0$ in equations (1.1) we obtain the simplified Hamiltonians

$$
\begin{aligned}
& h_{1}=\frac{1}{2}\left(p_{1}^{2}+p_{2}^{2}\right)+\frac{1}{8} \omega\left(4 x^{2}+y^{2}\right)-\frac{1}{2} x^{4}-\frac{3}{8} x^{2} y^{2}-\frac{1}{32} y^{4} \\
& h_{2}=\left(8 p_{2}\left(p_{1} y-p_{2} x\right)-x y^{4}-2 x^{3} y^{2}+2 \omega x y^{2}\right)^{2}
\end{aligned}
$$

and the system can be simplified further in this way:

$$
\begin{aligned}
& h_{1}=\frac{1}{2}\left(p_{1}^{2}+p_{2}^{2}\right)+\frac{1}{8} \omega\left(4 x^{2}+y^{2}\right)-\frac{1}{2} x^{4}-\frac{3}{8} x^{2} y^{2}-\frac{1}{32} y^{4} \\
& h_{2}=8 p_{2}\left(p_{1} y-p_{2} x\right)-x y^{4}-2 x^{3} y^{2}+2 \omega x y^{2}
\end{aligned}
$$

This system can be easily separated in several ways but we choose here to apply the method of the KCs for a double reason: first it gives us the opportunity the show how the method works in a very simple situation; second it provides the form of the functions $F$ and $G$ that will be generalized in (4.5) and, successively, in (5.3). 
The canonical equations can be written in this form

$$
\begin{aligned}
\dot{p_{1}} & =\frac{1}{4} x\left(8 x^{2}+3 y^{2}-4 \omega\right) \\
\dot{p_{2}} & =\frac{1}{8} y\left(6 x^{2}+y^{2}-2 \omega\right) \\
\dot{x} & =p_{1} \\
\dot{y} & =p_{2}
\end{aligned}
$$

and

$$
\begin{aligned}
p_{1}^{\prime} & =y^{4}+\left(6 x^{2}-2 \omega\right) y^{2}+8 p_{2}{ }^{2} \\
p_{2}^{\prime} & =4 x y^{3}+\left(4 x^{3}-4 \omega x\right) y-8 p_{2} p_{1} \\
x^{\prime} & =8 p_{2} y \\
y^{\prime} & =-16 x p_{2}+8 y p_{1}
\end{aligned}
$$

The syzygy of the equations of motion is easy to find:

$$
x^{\prime}=8 p_{2} y=8 y \dot{y}=\left(4 y^{2}\right)^{\bullet}
$$

which is equation (3.1) with

$$
F=x \quad G=4 y^{2}
$$

The syzygy left us with two fundamental functions verifying the condition $F^{\prime}=\dot{G}$.

The next step is to guess the form of the control matrix $M$. To this purpose, we make use of the linear conjecture and assume that the entries of the matrix have a linear expression in $F$ and $G .^{\text {a }}$ Replacing now (3.4) into equations (2.1) one finds, for example, this simple solution:

$$
\begin{array}{lll}
\alpha_{0}=0 & \alpha_{1}=2 & \alpha_{2}=0 \\
\beta_{0}=1 / 8 & \beta_{1}=0 & \beta_{2}=0 \\
\gamma_{0}=0 & \gamma_{1}=0 & \gamma_{2}=2 \\
\delta_{0}=0 & \delta_{1}=0 & \delta_{2}=0
\end{array}
$$

which gives us the control matrix

$$
M=\left(\begin{array}{cc}
2 F & 1 / 8 \\
2 G & 0
\end{array}\right)
$$

According to Theorem 2.3, the eigenvalues of $M$ are the separation coordinates of our problem:

$$
x \pm \sqrt{x^{2}+y^{2}} .
$$

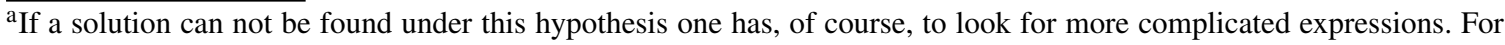
instance quadratic functions.
} 
Remark 4.1. We can eventually complete these coordinates in a canonical transformation:

$$
\begin{aligned}
X & =x+\sqrt{x^{2}+y^{2}} \\
Y & =x-\sqrt{x^{2}+y^{2}} \\
P_{1} & =\frac{p_{2} \sqrt{x^{2}+y^{2}}-p_{2} x+p_{1} y}{2 y} \\
P_{2} & =\frac{-p_{2} \sqrt{x^{2}+y^{2}}-p_{2} x+p_{1} y}{2 y}
\end{aligned}
$$

In order to get useful informations for the case $b=0$ (see next section), let's see how our functions $F$ and $G$ have to be modified to solve problem (4.1). The only difference in the equations of motion is that the coordinates of the vector field $X_{h_{2}}$ are now multiplied by the constant factor

$$
2\left(8 p_{2}\left(p_{1} y-p_{2} x\right)-x y^{4}-2 x^{3} y^{2}+2 \omega x y^{2}\right) .
$$

The form of equation (3.2) suggests to divide $F$ by the same constant factor. After adjusting the numerical coefficients, our functions take the form

$$
\begin{aligned}
& F=\frac{x}{8 p_{2}\left(p_{1} y-p_{2} x\right)-x y^{4}-2 x^{3} y^{2}+2 \omega x y^{2}} \\
& G=8 y^{2}
\end{aligned}
$$

These functions still verify equation $F^{\prime}=\dot{G}$ where the derivatives are now calculated with respect to the flows of the Hamiltonians in (4.1). Instead of proceeding as before with the linear conjecture it seems easier, in this case, to find a quadratic solution of (2.1). ${ }^{\mathrm{b}}$ This gives us the following matrix

$$
M=\left(\begin{array}{cc}
4 h_{2} F^{2}+G / 8 F / 8 \\
4 h_{2} F G & G / 8
\end{array}\right)
$$

As before, and according to Theorem 2.3, the eigenvalues of $M$ are the separation coordinates of our problem:

$$
2 x^{2}+y^{2} \pm 2 x \sqrt{x^{2}+y^{2}}
$$

Remark 4.2. The complete canonical transformation is

$$
\begin{aligned}
X & =2 x^{2}+y^{2}+2 x \sqrt{x^{2}+y^{2}} \\
Y & =2 x^{2}+y^{2}-2 x \sqrt{x^{2}+y^{2}} \\
P_{1} & =\frac{\left(-2 x p_{2}+y p_{1}\right) \sqrt{x^{2}+y^{2}}+2 x^{2} p_{2}-x y p_{1}+y^{2} p_{2}}{4 y^{3}} \\
P_{2} & =\frac{\left(2 x p_{2}-y p_{1}\right) \sqrt{x^{2}+y^{2}}+2 x^{2} p_{2}-x y p_{1}+y^{2} p_{2}}{4 y^{3}}
\end{aligned}
$$

${ }^{\mathrm{b}}$ See the last comment in Remark 7.1. 


\section{The degenerate case $b=0$}

Replacing $b=0$ in (1.1) we get:

$$
\begin{aligned}
& h_{1}=\frac{1}{2}\left(p_{1}^{2}+p_{2}^{2}\right)+\frac{1}{8} \omega\left(4 x^{2}+y^{2}\right)-\frac{1}{2} x^{4}-\frac{3}{8} x^{2} y^{2}-\frac{1}{32} y^{4}-\frac{a^{2}}{2 x^{2}} \\
& h_{2}=\left(8 p_{2}\left(p_{1} y-p_{2} x\right)-x y^{4}-2 x^{3} y^{2}+2 \omega x y^{2}\right)^{2}+16 a^{2}\left(y^{4}-4 \frac{p_{2}^{2} y^{2}}{x^{2}}\right)
\end{aligned}
$$

To find a syzygy from the equations of motion seems quite complicated in this case. Instead, we'll take advantage from the form of the functions $F$ and $G$ in (4.5) and we'll try to generalize them to the case $a \neq 0$. We can add a multiple of $a$ to the denominator and try something like:

$$
\begin{aligned}
F & =\frac{x+a f_{2}}{8 p_{2}\left(p_{1} y-p_{2} x\right)-x y^{4}-2 x^{3} y^{2}+2 \omega x y^{2}+a f_{1}} \\
G & =8 y^{2}+\frac{a g_{1}}{8 p_{2}\left(p_{1} y-p_{2} x\right)-x y^{4}-2 x^{3} y^{2}+2 \omega x y^{2}+a f_{1}}
\end{aligned}
$$

where $f_{1}, f_{2}$ and $g_{1}$ are functions of $\left(p_{1}, p_{2}, x, y\right)$. These functions $F$ and $G$ clearly reduce to the previous ones when $a=0$. Writing explicitly equation $F^{\prime}=\dot{G}$ one finds

$$
f_{1}=-\frac{8 p_{2} y}{x} \quad f_{2}=0 \quad g_{1}=-\frac{128 y\left(\left(-x p_{1}+a\right) y+2 x^{2} p_{2}\right)}{x}
$$

Replacing in $F$ and $G$ and simplifying, we are left with the following functions:

$$
\begin{aligned}
& F=-\frac{x^{2}}{x^{2} y^{4}+\left(2 x^{4}-2 \omega x^{2}\right) y^{2}+8 p_{2}\left(-x p_{1}+a\right) y+8 x^{2} p_{2}^{2}} \\
& G=8 y^{2}+\frac{128 a y\left(\left(-x p_{1}+a\right) y+2 x^{2} p_{2}\right)}{x^{2} y^{4}+\left(2 x^{4}-2 \omega x^{2}\right) y^{2}+8 p_{2}\left(-x p_{1}+a\right) y+8 x^{2} p_{2}^{2}}
\end{aligned}
$$

With these functions it's quite hard to solve equations (2.1) (especially if one has to use quadratic functions in $F$ and $G$ ) but here again we can take inspiration from the previous case. It's easy to check that the control matrix is here the same as in (4.6):

$$
M=\left(\begin{array}{cc}
4 h_{2} F^{2}+G / 8 F / 8 \\
4 h_{2} F G & G / 8
\end{array}\right)
$$

The eigenvalues of $M$ are separation coordinates for problem (5.1).

\section{The generic case}

The generic problem has been presented in page 347 :

$$
\begin{aligned}
& h_{1}=\frac{1}{2}\left(p_{1}^{2}+p_{2}^{2}\right)+\frac{1}{8} \omega\left(4 x^{2}+y^{2}\right)-\frac{1}{2} x^{4}-\frac{3}{8} x^{2} y^{2}-\frac{1}{32} y^{4}-\frac{a^{2}}{2 x^{2}}-\frac{b^{2}}{2 y^{2}} \\
& h_{2}=\left(8 p_{2}\left(p_{1} y-p_{2} x\right)-x y^{4}-2 x^{3} y^{2}+2 \omega x y^{2}+8 b^{2} \frac{x}{y^{2}}\right)^{2}+16 a^{2}\left(y^{4}-4 \frac{p_{2}^{2} y^{2}}{x^{2}}\right)
\end{aligned}
$$

The equations are now so complex than any attempts to generalise (5.3) adding unknown functions, as in (5.2), generate equations that Maple cannot solve. We need an easier guess. 
Let's take a look at $h_{2}$ and the denominator $D$ of $F$ and $G$ in the previous case $b=0$. $D$ contains all the terms in the first parentheses of $h_{2}$ multiplied by $x$ :

$$
\begin{aligned}
& D=x^{2} y^{4}+\left(2 x^{4}-2 \omega x^{2}\right) y^{2}+8 p_{2}\left(-x p_{1}+a\right) y+8 x^{2} p_{2}^{2} \\
& h_{2}=\left(8 p_{2}\left(p_{1} y-p_{2} x\right)-x y^{4}-2 x^{3} y^{2}+2 \omega x y^{2}\right)^{2}+16 a^{2}\left(y^{4}-4 \frac{p_{2}^{2} y^{2}}{x^{2}}\right)
\end{aligned}
$$

When we move to the generic problem, the only difference in $h_{2}$ is the appearance of an extra term $8 b^{2} x / y^{2}$ in the first parentheses. The easiest possible guess one can come up with at this stage, is to add the same term, multiplied by $x$, to $D$. After simplification this gives the functions

$$
\begin{aligned}
& F=-\frac{x^{2} y^{2}}{2 x^{4} y^{4}+\left(y^{6}-2 \omega y^{4}+8 y^{2} p_{2}^{2}-8 b^{2}\right) x^{2}-8 x y^{3} p_{1} p_{2}+8 a y^{3} p_{2}} \\
& G=8 y^{2}+\frac{128 a y^{3}\left(\left(-x p_{1}+a\right) y+2 x^{2} p_{2}\right)}{2 x^{4} y^{4}+\left(y^{6}-2 \omega y^{4}+8 y^{2} p_{2}^{2}-8 b^{2}\right) x^{2}-8 x y^{3} p_{1} p_{2}+8 a y^{3} p_{2}}
\end{aligned}
$$

Surprisingly enough these functions continue to verify the reduced KC: $F^{\prime}=\dot{G}$. A straightforward calculation proves that even the form of the control matrix is almost the same:

$$
M=\left(\begin{array}{cr}
4 h_{2} F^{2}+G / 8 & F / 8 \\
4 h_{2} F G+8192 a^{2} b^{2} F & G / 8
\end{array}\right)
$$

According to Theorem 2.3 the eigenvalues of $M$ are separation coordinates for the generic problem.

\section{Final remarks}

\section{Remark 7.1.}

- It is interesting to remark that, from the standpoint of the KCs, the generic problem is simply a natural generalisation of the degenerate case $b=0$. This generalisation is nothing more than the addition of the term $8 b^{2} x^{2} / y^{2}$ in the denominator of $F$ and $G$.

- This example shows, in our opinion, that the method of the KCs is a powerful tool in separating the coordinates of complex systems. The key-point is the determination of the functions $F$ and $G$, solutions of the reduced $K C$. This goal can be achieved analysing first the equations of motion in simple degenerate cases for a possible syzygy, and generalising these solutions to the more complicated cases.

- In order to guess the form of the entries of the control matrix $M$, one makes use of functions of $F$ and $G$ (whose coefficients are constants of the motion). The experience suggests that linear functions are, in general, possible. At least this is the case for all the other HénonHeiles systems that have been separated till now [9]. It would be interesting to know if such a linear solution exists even for this system. The separation coordinates would result significantly simplified. 
Remark 7.2. The 1:12:16 system can be written with 4 constants $\omega, a, b$ and $c$ [5], in a more general form than the one used by Conte, Musette and Verhoeven in [3]:

$$
h_{1}=\frac{1}{2}\left(p_{1}^{2}+p_{2}^{2}\right)+\frac{1}{8} \omega\left(4 x^{2}+y^{2}\right)-\frac{1}{2} x^{4}-\frac{3}{8} x^{2} y^{2}-\frac{1}{32} y^{4}+\frac{c\left(4 x^{2}+y^{2}\right)}{y^{6}}-\frac{a^{2}}{2 x^{2}}-\frac{b^{2}}{2 y^{2}}
$$

and

$$
\begin{aligned}
h_{2}= & \left(8 p_{2}\left(y p_{1}-x p_{2}\right)-x y^{4}-2 x^{3} y^{2}+2 \omega x y^{2}+8 b^{2} \frac{x}{y^{2}}-32 \frac{c x\left(2 x^{2}+y^{2}\right)}{y^{6}}\right)^{2} \\
& +16 a^{2}\left(y^{4}-32 \frac{c}{y^{4}}-4 \frac{p_{2}^{2} y^{2}}{x^{2}}\right)
\end{aligned}
$$

This system clearly reduces to (6.1) if $c=0$. The functions $F$ and $G$ can be easily modified adding an extra c-term in the same way we did in Section 6 for the b-term. The functions so obtained continue to verify the reduced KC but a solution of equations (2.1) seems hard to find either with linear or quadratic functions.

The 4 constant problem still remains unsolved.

\section{Acknowledgement}

This work was funded by Zayed University, Abu Dhabi, through the Startup Grant R16030.

\section{References}

[1] S. Baker, V. Z. Enolskii and A. P. Fordy, Integrable quartic potentials and coupled KdV equations, Phys. Lett. A, 201, 167174, 1995.

[2] M. Blaszak and S. RauchWojciechowski, A generalized HénonHeiles system and related integrable Newton equations, Journal of Mathematical Physics, 35, 1693-1709, 1994, http://dx.doi.org/ $10.1063 / 1.530565$

[3] R. Conte, M. Musette and C. Verhoeven. Explicit integration of the Hénon-Heiles Hamiltonians, Journal of Nonlinear Mathematical Physics, 12, 2005.

[4] R. Conte, M. Musette and C. Verhoeven. Painlevé property of the Hénon-Heiles Hamiltonians, Séminaires \& Congrès, 14, 65-82, 2006.

[5] J. Hietarinta, Direct method for the search of the second invariant, Phys. Rep. 147, 87154, 1987.

[6] F. Magri. The Kowalevski's top and the method of syzygies. Ann. Inst. Fourier, Grenoble 12, 21472159, 2005.

[7] F. Magri, T. Skrypnyk. The Clebsch System. https://arxiv.org/abs/1512.04872, 2015.

[8] V. Ravoson, A. Ramani, B. Grammaticos. Generalized separability for a Hamiltonian with nonseparable quartic potential. Phys. Letters A 191 9195, 1994.

[9] N. Sottocornola. Separation coordinates in Hénon-Heiles systems. To be published.

[10] C. Verhoeven. Integration of Hamiltonian systems of Hénon-Heiles type and their associated soliton equations. PhD thesis, Vrije Universiteit Brussel, May 2003.

[11] C. Verhoeven, M. Musette and R. Conte. Integration of a generalized Hénon-Heiles Hamiltonian, 2001, https://arxiv.org/abs/nlin/0112030.

[12] S. Wojciechowski, Separability of an integrable case of the Hénon-Heiles system, Phys. Lett. A, 100, $277278,1984$.

[13] S. Wojciechowski, Integrability of one particle in a perturbed central quartic potential, Physica Scripta $31,433438,1985$. 